\title{
Use Cases for 5G Networks Using Small Cells
}

\author{
Alexandros Kostopoulos ${ }^{1, *}$, Ioannis P. Chochliouros ${ }^{1}$, Daniele Munaretto ${ }^{2}$, Claudio \\ Meani $^{3}$, Claus Keuker ${ }^{4}$, Elisenda Temprado Garriga ${ }^{5}$, Javier Fernandez Hidalgo ${ }^{6}$, \\ Miguel Catalan $\mathrm{Cid}^{6}$, Hicham Khalife ${ }^{7}$, Fidel Liberal ${ }^{8}$ \\ 1, Hellenic Telecommunications Organization S.A. (OTE) \\ \{alexkosto, ichochliouros\}@oteresearch.gr \\ ${ }^{2}$ Athonet, ${ }^{3}$ Italtel, ${ }^{4}$ Smart Mobile Labs, ${ }^{5}$ Zodiac Inflight Innovations, ${ }^{6}$ i2CAT, ${ }^{7}$ Thales, \\ ${ }^{8}$ Universidad del Pais Vasco
}

\begin{abstract}
In this paper, we discuss the main use cases and scenarios we consider in the context of 5G ESSENCE project [1]. The first use case investigates edge network acceleration at a stadium. The second use case is focused on end-to-end slicing for mission critical applications. The third scenario considers in-flight communications and entertainment system.
\end{abstract}

Keywords: Cloud-enabled Small Cell (CESC); Mobile Edge Computing (MEC); Network Functions Virtualization (NFV); Radio Resource Management (RRM).

\section{Introduction}

5G ESSENCE [1] addresses the paradigms of Edge Cloud computing and Small Cell as-a-Service (SCaaS) by fuelling the drivers and removing the barriers in the Small Cell (SC) market, forecasted to grow at an impressive pace up to 2020 and beyond, and to play a "key role" in the 5G ecosystem. The 5G ESSENCE framework provides a highly flexible and scalable platform, able to support new business models and revenue streams by creating a neutral host market and reducing operational costs, by offering new opportunities for ownership, deployment, and operation.

As the telecom ecosystem moves towards the innovative 5G era, important performance factors such as end-to-end (E2E) latency critically depend on whether the mobile edge and the target applications reside in an edge cloud close to the user or not. Building upon these technological foundations [2]-[9], very ambitious objectives are targeted, culminating with the prototyping and demonstration of the 5G ESSENCE system in three real-life use cases associated to vertical industries, that is: (i) Edge network acceleration in a crowded event; (ii) mission critical applications for public safety (PS) communications providers, and; (iii) in-flight entertainment and connectivity (IFEC) communications. In this paper, we discuss 
the aforementioned use cases by considering the involved actors, per case, as well as the deployment topology.

\section{5G Edge Network Acceleration at a Stadium}

\subsection{Overall description}

Broadcasters are looking for new ways to cover events, to offer exciting point-ofview perspectives to viewers on one hand, and to reduce production and delivery costs on the other. At the same time, network operators target to increase the usage of their networks and stadium owners have a strong interest in making the visitors' experience as pleasant as possible, as well as to promote sponsors increasing siderevenues apart from ticket sales. The reception of live content from cameras located in the playing field, replays, and additional contextual information on mobile devices, is a strong use case for all the above actors. The main challenges even with $4 \mathrm{G}$ networks are the delay in the delivery of such content in the range of milliseconds rather than seconds, and the considerable strain imposed on the backhaul network. By deploying a 5G ESSENCE-like network, these challenges can be efficiently addressed.

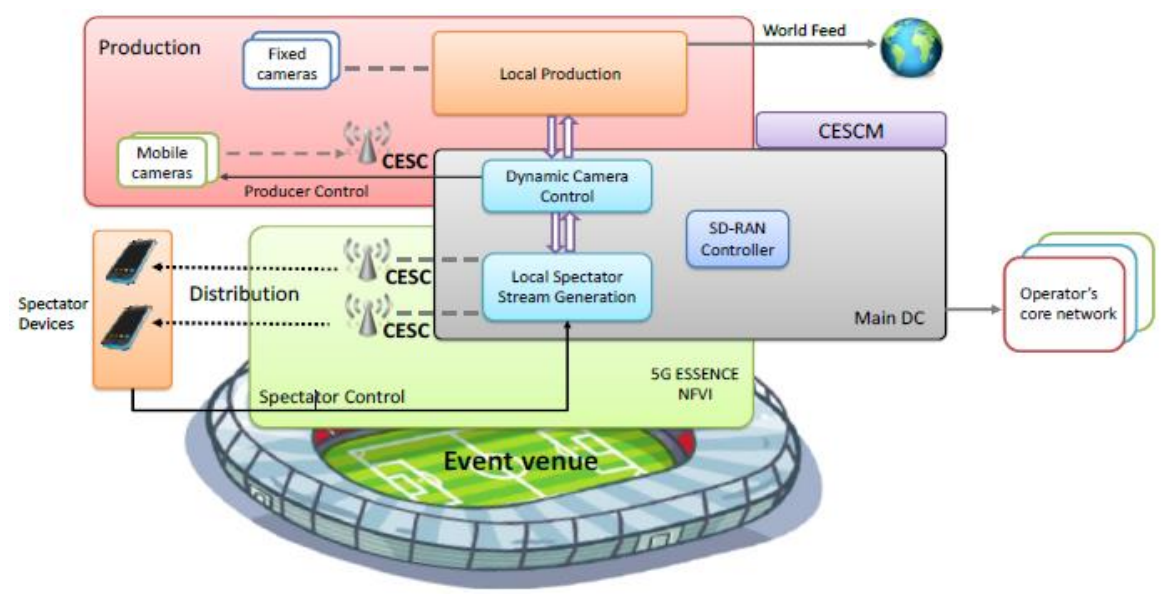

Fig. 1. 5G Edge Network acceleration at Stadium

5G ESSENCE delivers benefits to media producers and mobile operators as it enables them to offer a highly interactive fan experience and it optimises operations by deploying "key functionalities" at the edge, i.e., evolved Multimedia Broadcast Multicast Services (eMBMS) or local network services like real-time analytics 
together with multitenancy support by small cells. By leveraging the benefits of small cell virtualisation and radio resource abstraction, as well as by optimising network embedded cloud, it becomes possible to ease the coverage and capacity pressure on the multimedia infrastructure, and also to increase security since content will remain locally. Furthermore, additional benefits for the operators and the venue owners arise, such as: (a) lower latency, due to shortening the data transmission path; (b) maintained backhaul capacity, due to playing out the live feeds and replays locally that puts no additional strain on the backhaul network and upstream core network components. The aforementioned use case is illustrated in Figure 1.

\subsection{Actors involved}

The actors involved in this scenario are listed below:

- Small Cell Network Operator (SCNO): Owner of the infrastructure deployed in the stadium.

- Virtual Small Cell Operator (VSCNO): Users of the infrastructure available in the stadium to provide services to the end-users.

- End-Users (EUs): Users of the networking services.

- Mobile Operators (MOs): Responsible of bringing the network and communication services to the stadium.

- Service Provider (SPs): Companies providing some of the Virtual Network Functions to the SCNO. Examples of key service providers for this use case could be football/sport society, live event organizer, municipality, video/content provider.

- Spectrum Owner (SO): In the licensed spectrum case, a stadium that leases the spectrum from an operator or a mobile operator that offers a service.

- Mission Critical - Public Safety (MC-PS): Public or private organizations in charge of performing, when needed, mission critical actions for the public safety (video surveillance, monitoring, autonomous local network).

\subsection{Deployment topology}

5G ESSENCE will demonstrate a combined 5G-based video production and video distribution towards delivering benefits to both media producers and mobile operators, who will be able to offer enriched event experience to their subscribers. The production/distribution of locally generated content through the 5G ESSENCE platform, coupled with value-added services and rich user context, will enable secure, high quality and resilient transmission in real-time, thus ensuring minimal latency. 
In the context of the 5G ESSENCE infrastructure sharing support, each network operator will be in position to optimise his network usage, resulting in lower OPEX. Additionally, network operators will be able to rapidly deploy new services, to deliver directly to users higher Quality of Experience (QoE), and to offer "Content as-a-Service", increased bandwidth, and storage solutions to content providers and venue owners. The content providers will also benefit from reduced latency and improved user QoE by positioning content on mobile edge and, in addition, they can offer augmented services by leveraging the network information. Finally, the stadium owner will obtain additional benefits by leveraging the deployed 5G ESSENCE infrastructure and its functionalities, by capitalising live contents to spectators from many cameras, etc.

The scenario provides the logic for distributing the live video feeds received from the local production room to local spectators in a highly efficient manner. Different scales facilities can be used for the validation of the deployment topology. First, a small-scale facility as the municipal open swimming pool with a capacity of 500 spectators, secondly a medium-scale facility like the municipal indoor stadium (which is used for basketball, volleyball and handball games) with a capacity of 2,000 spectators and thirdly a large-scale facility as the municipal football stadium "Stavros Mavrothalasitis" which is located at the centre of Egaleo town (in Athens, Greece) and it is an open stadium with capacity of about 8,000 spectators.

Considering a proper selection from the aforementioned facilities, the selected facility will be covered with a cluster of multitenant, eMBMS enabled as CESCs (Cloud-enabled Small Cells) and, together with the CESCM (CSC Manager) and the Main DC (Data Centre), they will be connected to the core networks of multiple telecom operators. The video content from cameras will be sent for processing locally at the Edge DC (similar to the proposed use case by ETSI MEC [13]). Then the video streams will be broadcasted locally by using the CESCs. Spectators will be able to dynamically select between different offered broadcast streams. In this and in similar big event scenarios, massive data traffic will not affect nor overload the backhaul connection, as it will be produced, processed and consumed just locally (5G MEC scenario).

\section{5G E2E Slicing for Mission Critical Applications}

\subsection{Overall description}

Latest 3GPP releases address the key requirements expressed from the Public Safety (PS) domain for next generation broadband public safety networks [14]. Further improvements to the 3GPP standards to cope with mission critical 
communications requirements are being considered as a central topic in the 3GPP requirements study for 5G [15]. Regarding PS service delivery models, there is a clear trend towards different forms of network sharing models as opposed to building out dedicated PS networks. For example, the Emergency Services Network in the UK is going to use the RAN (Radio Access Network) infrastructure of a commercial provider; Blue Light Mobile service by PS operator Astrid in Belgium offers access through roaming agreements with commercial operators; FirstNet in the US, while it counts with spectrum dedicated to PS, is expected to enable secondary use by commercial applications. In this context, multitenancy becomes a cornerstone challenge at an international level.

5G ESSENCE common orchestration of radio, network and cloud resources is expected to significantly contribute to the fulfilment of the requirements of the PS sector, thus bringing new tools to share both radio and edge computing capabilities in localised/temporary network deployments between PS and commercial users. The challenge consists on allocating radio, network and cloud resources to the critical actors (e.g., the First Responders) who, by nature, require prioritised and high quality services.

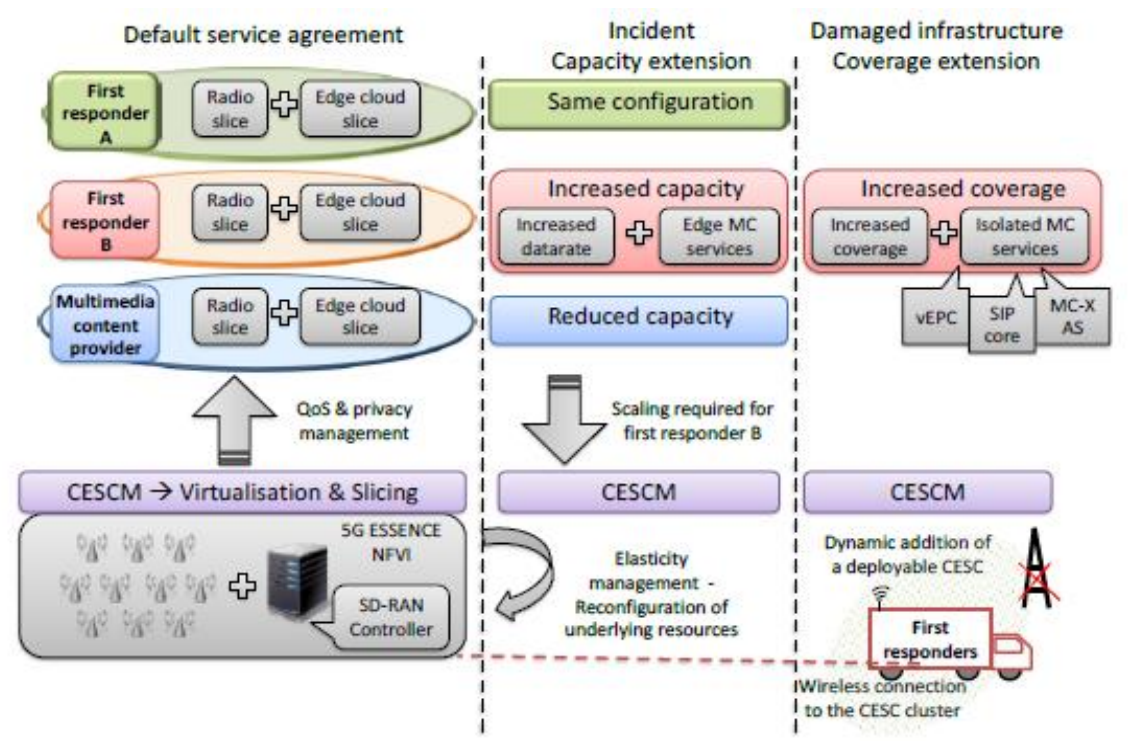

Fig. 2. Mission Critical applications for Public Safety

Indeed, the public safety operators are "shifting" their business model from a completely owned infrastructure model to one playing the role of a Mobile Virtual Network Operator (MVNO) between multiple parties owning and operating mobile networks and PS end-users. From a broader perspective, such deployment can be seen as a dedicated mission critical slice spanning across multiple operators 
domains. In such configuration, the relevant public safety operator "buys" connectivity to multiple legacy mobile operators and guarantees to his public safety customers connectivity, resilience and required quality of service (QoS) for PS operations. In practice, such approach has multiple benefits to PS operators, as discussed below.

First, it reduces the costs of buying, installing and maintaining dedicated infrastructures. Note that these physical infrastructures are not exploited to their optimal capacity. Second, the flexibility offered by such solutions enables (at least theoretically) MVNOs to adapt their offers to their customers. However, this approach comes at the price of pre-negotiated contracts between the MVNOs and multiple legacy providers, in order to ensure a high availability and a 'guaranteed" throughput for PS users.

For an allocation like this in order to be efficient and so to "enable" E2E network slicing, the 5G ESSENCE solution shall be applied. The aforementioned use case is schematically illustrated in Figure 2.

\subsection{Actors involved}

Demonstrating the 5G ESSENCE impact on mission critical deployments is performed with the following actors:

- A legacy mobile operator (Platform owner) offering its infrastructure to classical end-users as well as to Public Safety virtual operators. Note here that virtual operators can rely on multiple legacy operators for additional guarantee.

- A public safety operator that offers connectivity services with "strict" QoS guarantee to First Responders. Note that in our situation we can have multiple virtual operators each one offering a "separate" slice for a different First Responder agency.

- First Responder 1: firefighters are end-users exploiting the connectivity offered by public safety operator through a dedicated slice. For the sake of demonstration, firefighters will use Mission Critical Push-To-Talk (MCPTT) application for their communications.

- First Responder 2: paramedics are another set of end-users relying upon the same public safety operator or another slice coming from a different operator, in order to exchange chat messages as well as pre-registered pictures for situation assessment.

- Legacy end-users constitute classical user that have subscribed to the legacy operator communication and Internet data offers. They are not part of any first responder entity but exploit only the network of the legacy mobile operator without any intermediary. 


\subsection{Deployment topology}

The 5G ESSENCE innovative platform will involve one -or more- PS communications providers that will use the resources offered by a deployed $5 \mathrm{G}$ ESSENCE platform for the delivery of communication services to PS organisations in a country/region. The 5G ESSENCE platform can be owned by, either a mobile (potentially virtual) network operator or even by a venue owner, such as in the Use Case 1. In the mission critical use case, the infrastructure owner will exploit the 5G ESSENCE system's capabilities to provide the required network/cloud slicing capabilities with dedicated Service Level Agreements (SLAs) to different types of tenants, thus prioritising the PS communications providers.

The corresponding Mission Critical (MC) use case will be organised in three main stages, described as below:

Stage 1: Under normal circumstances, the 5G ESSENCE platform owner is providing the required network slices to different tenants. Each network slice is composed of an allocated data rate over a coverage area (which is mapped by the cSD-RAN (centralised Software-Defined RAN) Controller to a portion of CESC radio resources) and an allocated of cloud resources (which is mapped to processing power/storage capabilities in the Edge DC). For the service of Public Safety organisations, normal operations require a certain amount of access capacity and communications features (e.g., group communications capabilities) supported in the area of the CESC cluster. This requirement will be "mapped" to a number of radio Key Performance Indicators (KPIs) in the CESCs and the deployment of Group Communication service instances at the edge for multimedia and missioncritical Application Servers (AS) for voice with enhanced responsiveness. In addition to the QoS guarantees for each tenant, the deployment owner has to assure the required levels of isolation in the provisioning of the network slices.

Stage 2: In the case where there is an emergency in the area, the CESCM will be able to react to the new service requirements. The PS communications provider may require additional service in order to "cope with" an increased number of First Responders or additional types of services, such as mission-critical video transmissions. Based on pre-arranged or on-demand service scaling policies, the CESCM will implement new elastic resource allocation schemes, giving priority access to First Responders and taking into account both radio (for the access connections) and cloud resources (for deploying more resource-consuming edge services). The deployment of edge service instances serves a two-fold objective: first, it enables close-to-zero delay in the mission-critical services; second, it allows maintaining the operability, even when the backhaul connection is damaged.

Stage 3: In case that ICT infrastructure is damaged during a natural disaster or a terrorist attack, the first action should "address" the need for radio coverage extension. In this stage, a deployable system to mitigate the damage in the macrobase stations, will be used. In the proposed use case, the deployable system will 
offer 5G connectivity to the First Responders in the field, consolidating the interoperability requirements. In order to "better orchestrate" the radio transmissions, the deployable system will be considered as a new CESC that can be dynamically integrated to the small cell cluster. In this way, the enhanced 5G ESSENCE SON (Self-Organising Networks) and RRM (Radio Resources Management) features can be applied to the coverage extension unit. The interconnection of the deployable unit with the CESC cluster will be made through a wireless backhauling technology.

\section{$4 \quad 5 G$ In-flight Communications and Entertainment System}

\subsection{Overall description}

Some years ago, only a single movie was available for all passengers to watch during a flight. Next, on-demand viewing options came, built onto the seats, where viewers could choose from an array of available television shows, movies and specials. Today, passengers bring their own devices on selected airlines. Thus, the wireless Inflight Entertainment (IFE) and broadband mobile connectivity increasingly have become actual commodity expectations both from airlines and passengers.

With the increasing demand for wireless IFE and passenger Bring-Your-OwnDevices (BYOD) connectivity, together with the demand for on board broadband mobile Internet connectivity, an important focus is laid on the on-board infrastructure. Current in-cabin wireless systems mainly rely on Wi-Fi technologies for the wireless IFE and broadband Internet connectivity (with backhaul connectivity offered through satellite links). Such solutions have several limitations such as prohibitive costs to the service providers, inability to support broadband cellular services, lack of flexibility for airlines operators, etc.

In order to offer cost-effective mobile broadband Internet connectivity, it is imperative to integrate on-board a neutral host solution that will allow multioperator connectivity to the passengers, also accounting for variable service offerings. Such host-neutral services are important for European airliners, since they traverse several regional boundaries served by a large variety of mobile operators. Regarding backhauling, terrestrial direct-air-to-ground (continental airspace) and satellite (oceanic airspace) solutions can used.

The unique architecture proposed in 5G ESSENCE (as schematically depicted Figure 3) which combines efficiently the virtualised and multi-tenant small cell networks with a multi-tier cloud edge infrastructure, is an essential and innovative step towards establishing a pioneering integrated In-Flight Entertainment and Connectivity (IFEC) system that will jointly deliver the required communication 
and network infrastructure for the wireless IFEC (to both the embedded IFE devices and the wireless BYODs).

Two different scenarios are envisaged in the corresponding IFEC use case. The first one consists of streaming and encoding to PErsonal Devices (PEDs) and aircraft devices (i.e., seat built-in screens). The purpose is about exploiting the Multi-Operator Core Network (MOCN) from 5G ESSENCE and LTE technologies so that to showcase multi-tenancy. That way, the Small Cell Network Operator -for instance the airline- can provide its proprietary IFEC services to the EUs (passengers) while leasing, at the same time, the same infrastructure to different VSCNOs, who will re-use it to give access to the same EUs to different services, such as Netflix, Spotify, etc., provided by themselves or by external Service Providers (SPs). For this concrete scenario, it is planned to "add" LTE to the current on-board $\mathrm{Wi}-\mathrm{Fi}$ architecture, in order to provide multiple services from different operators/providers at the same time and in the same network infrastructure.

The second scenario consists upon providing efficient wireless multicast onboard. Networking speaking, the cabin of an aircraft is a super "dense" scenario. As mentioned above, all wireless communications on-board are currently done via $\mathrm{Wi}$ Fi technologies, which actually "face" many limitations, in particular when many devices "share" the same medium. It becomes even more challenging, when it is planned to wirelessly multicast the same content at high-data rates to multiple PEDs and aircraft devices (for instance, video at high resolution). Thus, this specific scenario aims at dealing with and solving this problem by combining the Evolved Multimedia Broadcast Services (eMBMS) from LTE with the 5G ESSENCE architecture for on-board applications. This way, the SCNO can lease his infrastructure to VSCNOs and SPs, so that all of them can simultaneously stream their feeds (for instance, sport events, documentaries, or TV soap operas). Like that, the passengers (EUs) can watch live TV content in high resolution while, flying to a new destination.

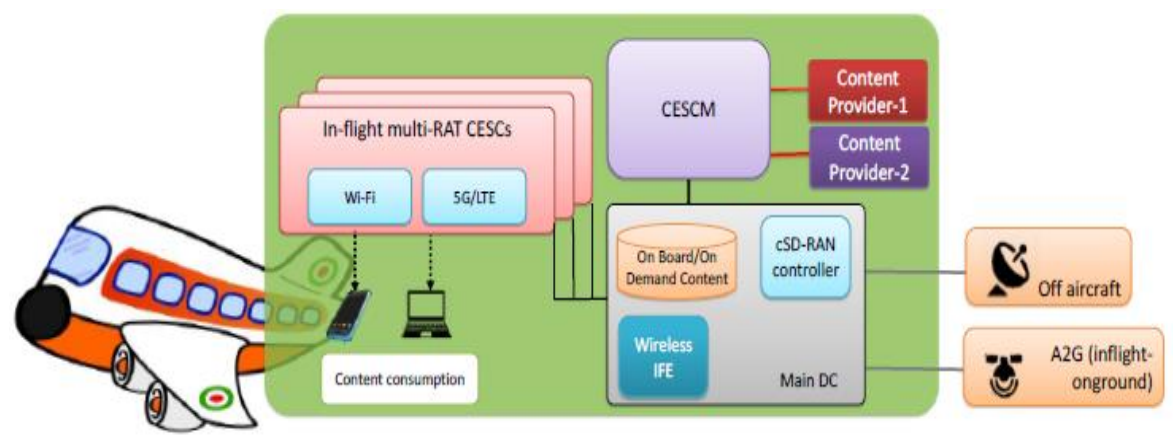

Fig. 3. Integrated In-flight Connectivity and Entertainment Systems 


\subsection{Actors involved}

The actors involved in the IFEC scenario are listed below:

- Small Cell Network Operator (SCNO): Owner of the infrastructure deployed in the aircraft. It can be the airline flying the aircraft or a network operator/content provider, who is interested in providing its services on-board.

- Virtual Small Cell Network Operator (VSCNO): Users of the infrastructure available in the aircraft to provide services to the end-users.

- End-Users (EUs): Users of the networking services provided by the VSCNO and SCNO (a.k.a. flight passengers).

- Mobile Operators (MOs): Responsible of bringing the network and communication services to the aircraft.

- Service Provider (SP): Companies providing some of the Virtual Network Functions to the SCNO.

- Spectrum Owner (SO): In the licensed spectrum case, it is the airline who leases the spectrum from an operator or a mobile operator that offers a service on-board (who provides the spectrum for free or for a charge).

\subsection{Deployment topology}

The 5G ESSENCE IFEC demo will test and validate the multi-tenancy enabled network solution for passenger connectivity and wireless broadband experience. The multi-RAT (Radio Access Technologies) CESCs will be implemented as a set of integrated access points to be deployed on-board. Afterwards, since IFE has to consider the explosive growth of multi-screen content consumption, the $5 \mathrm{G}$ ESSENCE CESCs will stream on-demand multi-screen video content (both from on-board 5G Edge DC servers and via satellite/air-to-ground links) to the wireless devices. The 5G ESSENCE CESCs will rely on broadcast links, in order to optimise the bandwidth usage.

Following the specific requirements for future IFEC solutions, as also currently outlined by various airlines and service providers, this 5G ESSENCE prototype platform will be tailored for a mock cabin deployment. The IFEC demonstration will be performed over a mock-up aircraft communications testbed installed, with core network connectivity to be incorporated both as on-board option and as a remote option steered via an emulated satellite link.

The use case will demonstrate the multitenant CESC for on-board connectivity and the Edge DC capabilities for hosting airborne applications (such as video player applications and files that can be made available for in-flight streaming) and caches (a version of the in-flight portal - gateway with which passengers can connect to). 
The video encoding and packaging service will support a wide range of input file formats, allowing easy integration with existing production systems, and a comprehensive array of output formats. Output parameters will be configurable, enabling precise tuning for the desired output devices.

The topology foreseen for this use case aims at combining the current on-board topology with the one provided by the 5G ESSENCE project. Currently, an aircraft is connected to the Internet via a broadband antenna which, at the same time, is connected an on-board server, which is connected to the multiple access points deployed around the cabin to provide the IFEC services to the clients.

The 5G ESSENCE architecture is be added to the current cabin topology as an additional server, which is connected to the broadband access and all the other onboard servers. This new server will contain 5G ESSENCE elements, such as the MOCN, the small cell manager or the EPC (Evolved Packet Core) on-board. The current access points are going to be replaced by small cells, which will also be connected to this 5G ESSENCE server. This way, they are going to provide the different services/applications to the on-board screens and PEDs.

\section{Discussion}

In this paper, we have discussed the main use cases in the context of the $5 \mathrm{G}$ ESSENCE actual research framework. The first use case is a $5 \mathrm{G}$ edge network acceleration at a stadium. In particular, 5G ESSENCE will demonstrate a combined 5G-based video production and video distribution towards delivering benefits to both media producers and mobile operators, who will be able to offer enriched event experience to their subscribers. The second use case is $5 \mathrm{G}$ end-to-end slicing for mission critical applications. In particular, 5G ESSENCE will involve one or more PS communications providers that will use the resources offered by a deployed 5G ESSENCE platform for the delivery of communication services to PS organisations in a country/region. Our third scenario is focused upon in-flight communications and entertainment system. This demo will test and validate the multi-tenancy enabled network solution for passenger connectivity and wireless broadband experience.

For each use case, we investigated the involved actors, as well as the deployment topology. As a next step, we intend to focus upon the evaluation process, as well as upon the extraction of the main technical and non-technical requirements. Part of this work is already presented in [10]. Apart from the evaluation process, 5G ESSENCE will consider market and business aspects, since its architecture allows the sharing of existing and new infrastructure by many operators in a multitenant environment. Thus, it will enable new business models that will help new entrant market players to develop and analyse the perspectives of potential "win-win" strategies, based on the developed solutions ([11], [12]). 
Acknowledgements. The paper has been based on the context of the 5G-PPP phase 2 " $5 \mathrm{G}$ ESSENCE" ("Embedded Network Services for 5G Experiences") Project (GA No.761592), funded by the European Commission.

\section{References}

1. 5G ESSENCE H2020 5G-PPP Project, http://www.5g-essenceh2020.eu.

7. Chochliouros, I.P., Giannoulakis I., Kourtis, et al:: A Model for an Innovative 5Goriented Architecture, based on Small Cells Coordination for Multi-Tenancy and Edge Service. In Proceedings of AIAI-2016, IFIP AICT 475, pp.666--675. Springer International Publishing Switzerland (2016)

3. L. Goratti, et al. (2016): Network Architecture and Essential Features for 5G: The SESAME Project Approach. In Proceedings of AIAI-2016, IFIP AICT 475, pp.676685. Springer International Publishing Switzerland (2016)

4. SESAME 5G-PPP Project (GA No.671596): Deliverable 2.2 ("Overall System and Architecture") (2016). Available at: http://www.sesame-h2020-5gppp.eu/Deliverables.aspx

5. SESAME 5G-PPP Project (GA No.671596): Deliverable 2.3 ("Specification of the CESC Components - First Iteration”) (2016)

6. SESAME 5G-PPP Project (GA No.671596), Deliverable 3.1 ("CESC Prototype design specifications and initial studies on Self-X and virtualization aspects") (2016). Available at: http://www.sesame-h2020-5g-ppp.eu/Deliverables.aspx

7. SESAME 5G-PPP Project (GA No.671596), Deliverable 2.4 ("Specification of the Infrastructure Virtualisation, Orchestration and Management") (2016)

8. Giannoulakis, I., Fajardo, J.O., et al.: Enabling Technologies and Benefits of MultiTenant Multi-Service 5G Small Cells. In Proceedings of the EuCNC-2016, pp.1--5

9. Costa-Perez, X., Swetina, J., Guo, T., Mahindra, R., and Rangarajan, S.: Radio Access Network Virtualization for Future Mobile Carrier Networks. IEEE Communications Magazine, 51(7), 27-35 (2013)

10. Chochliouros, I.P., Kostopoulos, A., Giannoulakis, I., Spiliopoulou, A.S., Belesioti, M., Sfakianakis, E., Kourtis, A., and Kafetzakis, E.: Using Small Cells from enhancing 5G Networks. In Proceedings of IEEE Conference on Network Function Virtualisation and Software-Defined Networks (NFV-SDN'17), pp.1--6. IEEE (2017)

11. Chochliouros, I.P., Sfakianakis, E., Belesioti, M., Spiliopoulou, A.S., and Dardamanis, A.: Challenges for Defining Opportunities for Growth in the 5G Era: The SESAME Conceptual Model. In Proceedings of the EuCNC-2016, pp.1--5 (2016)

12. Khan, G.A., et al.: Network sharing in the next mobile network: TCO reduction, management flexibility, and operational independence. IEEE Communications Magazine, 49(10), 34-142 (2011)

13. European Telecommunication Standards Institute (ETSI): http://www.etsi.org/technologies-clusters/technologies/multi-access-edge-computing

14. The Third Generation Partnership Project (3GPP): http://www.3gpp.org/newsevents/3gpp-news/1455-Public-Safety

15. The Critical Communications Association (TCCA): 4G and 5G for Public Safety Technology options (White Paper). Cambridgeshire, UK (2017) 